\title{
Protótipo de dispositivo facilitador para digitalização 3D por fotogrametria com smartphones
}

Prototype of a facilitating device for photogrammetric 3D acquisition with smartphones

\author{
Paulo Victor de Farias Dantas \\ Universidade Federal do Rio Grande do Sul, Brasil \\ victor.dantas@ufrgs.br \\ Thiago Rafael Rodrigues Ribeiro \\ Universidade Federal do Rio Grande do Sul, Brasil \\ thiago.ribeiro@ufrgs.br
}

\author{
Underléa Miotto Bruscato \\ Universidade Federal do Rio Grande do Sul, Brasil \\ underlea.bruscato@ufrgs.br \\ Fabio Pinto da Silva \\ Universidade Federal do Rio Grande do Sul, Brasil \\ fabio.silva@ufrgs.br
}

\begin{abstract}
This paper presents the prototyping of a facilitating device for photogrammetric 3D acquisition with smartphones. It aims to deliver basic photogrammetry knowledge and a low-cost do-it-yourself alternative to commercial 3D scanning systems, consisting of interlocked laser cut parts and free third-party software. The proposed device was tested, and the resulting 3D model was qualitatively compared to those obtained by a handheld photogrammetry approach, a conoscopic laser scanning device, and a portable structured-light scanning device. The proposed device provides camera stabilization and homogenous distribution of image acquisition which result in improved 3D reconstruction quality, compared to a handheld approach.
\end{abstract}

Keywords: Digital fabrication;3D scanning; Close-range photogrammetry; Fab Lab; Facilitating devices.

\section{Introdução}

Tecnologias de fabricação digital vêm se tornando cada vez mais populares e alcançando um número crescente de novos usuários e aplicações, redefinindo relações de consumo e produção. Até pouco tempo estes recursos ainda eram restritos a algumas áreas como a produção industrial, construção civil e medicina. Lipson e Kurman (2013) explicam que rupturas tecnológicas ocorrem quando pessoas comuns ganham acesso a novas ferramentas, e as aplicam na solução de problemas de seu cotidiano.

A impressão 3D - nome popularmente dado a um conjunto de técnicas de manufatura aditiva -, por exemplo, foi desenvolvida ainda na década de 1980 (Gershenfeld, 2012; Lipson \& Kurman, 2013), e até ganhar o interesse popular nesta última década, era empregada de forma discreta na produção em pequena escala de alguns componentes.

O interesse popular por tecnologias de impressão 3D provocou um crescimento ímpar neste mercado, estimulando o desenvolvimento de novas tecnologias e serviços, buscando atender a todos os níveis de novos consumidores. Lipson e Kurman (2013) mostram que em apenas 5 anos, de 2007 a 2011, o número de impressoras 3D pessoais vendidas superou o de equipamentos similares de nível industrial.

Além disso, são nas aplicações cotidianas de usuários comuns, profissionais independentes, e pequenos grupos de pesquisa, que a impressora 3D está chegando perto de fazer justiça à fama que Ihe foi dada - "a máquina capaz de fabricar (quase) tudo" (Gershenfeld, 2012; Lipson \& Kurman, 2013).
De forma semelhante, outras ferramentas de fabricação digital, como corte a laser e usinagem CNC, vêm ganhando espaço entre usuários comuns e pequenos empreendedores, criando novos produtos e serviços, e estimulando avanços nessas áreas também. Para Gershenfeld (2012), a revolução da fabricação digital não está na oposição entre métodos aditivos e subtrativos de manufatura, mas sim na habilidade de transformar dados em objetos físicos, e objetos físicos novamente em dados.

Neste sentido, este estudo tem como objetivo fornecer conhecimentos e ferramentas que auxiliem a transformação de objetos físicos em dados. Para este propósito apresentam-se diretrizes para a criação de um dispositivo facilitador para a digitalização tridimensional por fotogrametria. O desenvolvimento foi parte de um workshop realizado no Laboratório de Inovação e Fabricação Digital da Escola de Engenharia, da Universidade Federal do rio Grande do Sul (LIFE/UFRGS), sob tema 'Fab Labs'.

São apresentados os princípios fotogramétricos que nortearam a configuração do dispositivo facilitador, o processo de prototipagem, e uma breve análise comparativa entre o resultado obtido com o dispositivo proposto de baixo custo e digitalizadores comerciais.

\section{Fab Lab}

A popularização de recursos de fabricação digital veio acompanhada da expansão da filosofia Do-It-Yourself (faça você mesmo), e de seu desdobramento tecnológico, mais focado na adaptação e criação de dispositivos eletrônicos, o movimento Maker (Lipson \& Kurman, 2013). Fab Labs são a materialização destes fenômenos, espaços de criação coletiva que impulsionam a inovação realizada pelos próprios 
usuários; atuando frequentemente nas esferas sociais e educacionais, empoderando seus participantes através da tecnologia e informação.

Um Fab Lab (abreviação do termo em inglês fabrication laboratory) é um espaço colaborativo de criação e de prototipagem rápida de objetos e produtos. O público alvo são designers, artistas, estudantes, makers, hakers e empreendedores, que almejam praticar, executar e melhorar seus conhecimentos em eletrônica, CAD/CAM e prototipagem.

A infraestrutura é composta de maquinário de comando numérico computadorizado ( $\mathrm{CNC})$ de nível profissional, porém de baixo custo, como máquinas de corte a laser, de corte de vinil, fresadora de alta precisão, impressora 3D e componentes eletrônicos. Este 'kit padrão' de máquinas é comum a todos Fab Labs, de sorte que processos e projetos desenvolvidos em um laboratório possam ser facilmente replicados em qualquer outro Fab Lab da rede. Para tanto, esses projetos necessitam de documentação para que possam ser compartilhados a fim de fortalecer a comunidade e levar adiante seus princípios educacionais de sensibilização à fabricação digital e pessoal, bem como democratização do conhecimento e do acesso aos meios modernos de fazer coisas (Eychenne \& Neves, 2013; Gershenfeld, 2012).

Segundo Eychenne e Neves (2013) "os Fab Labs se inscrevem nos mecanismos de trabalho colaborativo da internet, onde são favorecidos e encorajados a troca e colaboração através de práticas inovadoras ascendentes e comunitárias, democratizando as ferramentas, permitindo que o usuário se transforme em 'ator' do processo." "Afinal, a verdadeira força de uma Fab Lab não é técnica; é social. São as pessoas inovadoras que impulsionam a economia do conhecimento" (Gershenfeld, 2012).

Em um curso criado por Gershenfeld (2006) intitulado 'How to Make Almost Anything' (Como fazer quase tudo, grifo nosso), foi identificado como resultado de maior peso a fabricação individual - útil para fabricar o que é único -, algo inexistente no mercado atualmente, onde as pessoas podem ainda localmente desenvolver soluções para problemas locais, valores compartilhados com os Fab Labs.

Hoje, com a fabricação para o mercado de uma pessoa, a cornucópia de máquinas que fazem outras máquinas agora se concentra em produzir produtos personalizados, que contenham sensações e experiências de seu criador. $E$ não apenas, há algo que vai além, uma vez que um dos objetivos da fabricação pessoal é exatamente produzir aquilo que as lojas não podem oferecer.

Uma consequência da relação inversa entre quantidade e qualidade na produção industrial é percebida pelo desperdício e produção de lixo, onde produtos são descartados muito antes do fim de sua vida útil, de forma que, sendo tão fácil se livrar das coisas, nos dessensibiliza dos produtos/objetos que temos (Sennett, 2008). A fabricação individual, por outro lado, retoma essa sensibilização quanto aos bens produzidos, uma vez que tanto a produção quanto seu produto estão intrinsecamente ligados àquele que o concebe, podendo ainda influenciar positivamente nesta famigerada produção de resíduos.

O desenvolvimento de projetos e fabricação de novos objetos nos Fab Labs passa muitas vezes pelo estudo de produtos e componentes físicos existentes, ou ainda, pela tomada de medidas (antropometria) da pessoa a qual estes se destinam. Para estas atividades, as tecnologias de digitalização tridimensional são imprescindíveis. Ainda assim, equipamentos para este fim não fazem parte do conjunto padrão de máquinas presentes em Fab Labs. Com vista a propor soluções para viabilização da digitalização tridimensional nesses ambientes, é demonstrada a possibilidade de desenvolvimento de um dispositivo facilitador conforme resultados apresentados neste artigo.

\section{Digitalização Tridimensional}

A digitalização tridimensional é processo através do qual atributos da superfície de um objeto físico são convertidos em dados. Os dados primários obtidos de uma digitalização tridimensional são chamados de nuvem de pontos; um conjunto de pontos de coordenadas (XYZ) distribuídos no espaço, podendo conter ainda outras informações, como cor (RGB). A partir de nuvens de ponto pode-se construir modelos virtuais da superfície digitalizada (Figura 1). a)

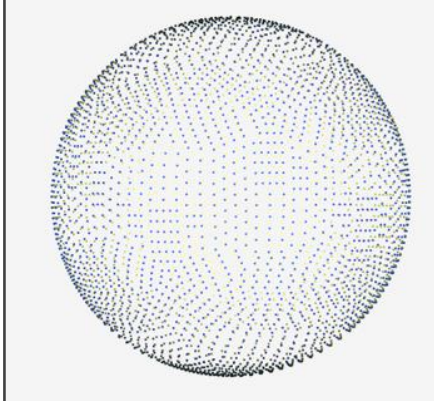

b)

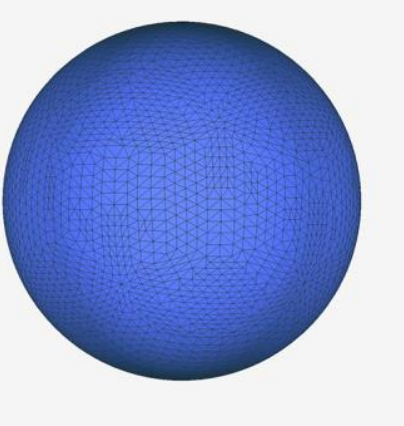

Figura 1: a) objeto esférico representado por uma nuvem de pontos b) modelo virtual de superfície poligonal.

Modelos obtidos por digitalização tridimensional podem servir para realizar medições, analisar superfícies, fabricar moldes, ou ainda podem ser aplicados com fidelidade a produtos inovadores (Silva, 2011). A digitalização tridimensional favorece o desenvolvimento de produtos personalizados. No entanto, apesar de sistemas de alta precisão virem se popularizando, eles ainda representam um grande investimento, dificultando sua utilização em projetos de baixo custo (Silva, 2011).

Tecnologias de digitalização tridimensional podem ser divididas entre aquelas em que a medição se faz por contato na superfície do objeto físico e aquelas que a fazem sem contato. As tecnologias sem contato são capazes de adquirir 
um grande volume de dados em um tempo relativamente curto e possibilitam a digitalização de objetos delicados ou difíceis de manusear.

Em estudos comparativos, Silva (2011) demonstra a precisão obtida com a utilização de sistemas comerciais a laser sem contato, equipamentos que, no entanto, podem custar algumas dezenas de milhares de reais (Figura 2). Como alternativa promissora, o autor destaca a técnica de fotogrametria (com os aplicativos 123D Catch e PhotoModeler Scanner), a qual representa um baixo custo de implementação, é adaptável à diversas situações, e apresenta precisão satisfatória para aplicações gerais. $\mathrm{Na}$ discussão dos resultados, o dispositivo facilitador será comparado a alguns desses sistemas.

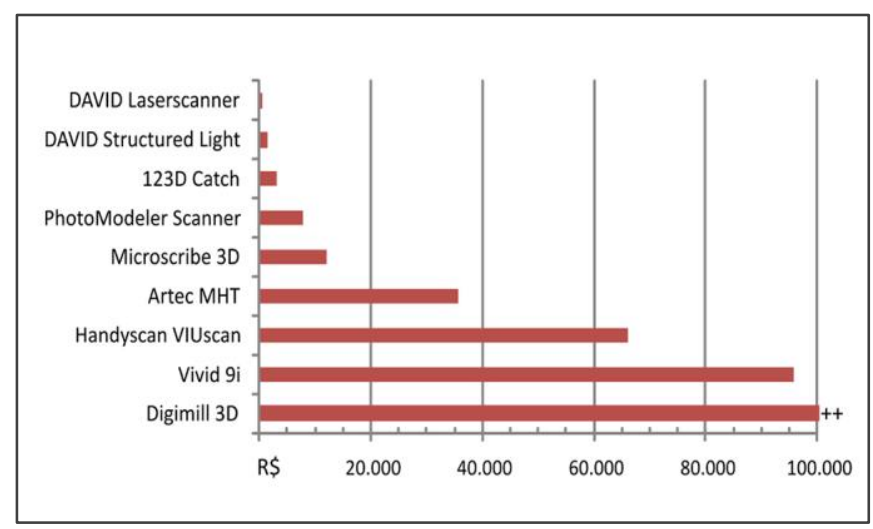

Figura 2: Custo estimado dos sistemas analisados por Silva (2011).

A seção seguinte trata da Fotogrametria e seus princípios de funcionamento. Estes conhecimentos serviram de base para o desenvolvimento do dispositivo facilitador para a digitalização proposta neste artigo.

\section{Fotogrametria}

A Fotogrametria é definida como a ciência, e técnica de interpretar e avaliar forma, dimensão e posição de objetos, a partir da análise e medição de imagens em que estes estão contidos (Foster \& Halbstein, 2014; Kasser \& Egels, 2002; Linder, 2009; Redweik, 2013). Originalmente é parte da Geodésia - ciência que estuda dimensões, forma e campo de gravidade da Terra - e pertence ao campo do sensoriamento remoto, que reúne um conjunto de técnicas destinadas a obtenção remota de informações sobre pontos na superfície da terra.

Com mais de um século de história as tecnologias empregadas na Fotogrametria evoluíram radicalmente, mais recentemente marcadas pela invenção do computador e desenvolvimentos no campo de visão computacional (Linder, 2009; Redweik, 2013). Desta forma, passou de dispositivos puramente ótico-mecânicos operados por especialistas, a processos inteiramente digitais e intuitivos.

Historicamente, a Fotogrametria tem sido aplicada principalmente na cartografia, com a finalidade de gerar modelos tridimensionais da superfície da terra, a partir dos quais é possível extrair curvas de nível, entre outros dados, para a geração de mapas detalhados. Neste processo, utilizam-se, geralmente, imagens fotográficas tomadas perpendicularmente ao solo por uma câmera montada em uma aeronave, que sobrevoa a área de interesse em grande altitude. Este tipo de aplicação fotogramétrica é uma variação da Fotogrametria a qual se dá 0 nome 'Fotogrametria Aérea'. Outra variação da técnica é denominada 'Fotogrametria Terrestre' ou 'Fotogrametria à curta distância'. Esta distingue-se da 'Fotogrametria Aérea' pelo fato de as imagens serem adquiridas paralelamente à superfície terrestre, à uma distância reduzida do objeto de interesse (Kasser \& Egels, 2002; Linder, 2009; Redweik, 2013; Tommaselli, Silva, Hasegawa, Galo, \& Dal Poz, 1999).

A aplicação fotogramétrica prevista neste artigo digitalização de pequenos artefatos utilizando smartphones e a estrutura facilitadora proposta - se enquadra na definição de fotogrametria à curta distância. Linder (2009), no entanto, frisa que apesar da distinção na nomenclatura, os princípios matemáticos e técnicos que regem o processo fotogramétrico são similares em ambos os casos.

Atualmente, a Fotogrametria à curta distância encontra-se em plena difusão, e vem sendo adotada em aplicações das mais diversas áreas, como: na preservação e documentação do patrimônio histórico e cultural, no monitoramento de deformações em edificações e terrenos, na indústria automotiva, aérea e náutica, em estudos forenses, na medicina dentária e ortopédica, em aplicações antropométricas, entre outras (Redweik, 2013).

Além de modelos tridimensionais da superfície da terra e mapas, os produtos da Fotogrametria podem ser modelos tridimensionais completos de artefatos, construções, indivíduos e objetos da natureza. Publicações voltadas a estes tipos de aplicações podem ser encontradas em periódicos das mais diversas áreas (Eulitz \& Reiss, 2015; Evin et al., 2016; Martínez, Ortiz, \& Gil, 2015; Pesce, Galantucci, Percoco, \& Lavecchia, 2015; Tang, Tang, \& Tay, 2016; Urbanová, Hejna, \& Jurda, 2015).

\section{Princípios de Fotogrametria}

A digitalização tridimensional por fotogrametria dá-se através da restituição estereoscópica. Este é o mesmo princípio que possibilita ao humano, e outros animais, a percepção espacial. Qualquer objeto tridimensional que estiver visível em ambas as vistas de um par estereoscópico, como nossos olhos, pode ser reconstruído tridimensionalmente (Kasser \& Egels, 2002). Como reconstrução entende-se aqui a determinação de suas coordenadas, isto é, posicionamento no espaço tridimensional.

As coordenadas são obtidas por triangulação, isto é, tomando como base a projeção de pontos no espaço sobre planos de imagem bidimensionais (Cuypers et al., 2009). Triangulando-se diversos pontos localizados em duas ou mais imagens é possível determinar a posição a partir de qual a imagem foi tirada, também chamada de orientação 
externa da imagem, e restituir a cena tridimensional que originou as imagens (Figura 3 ).

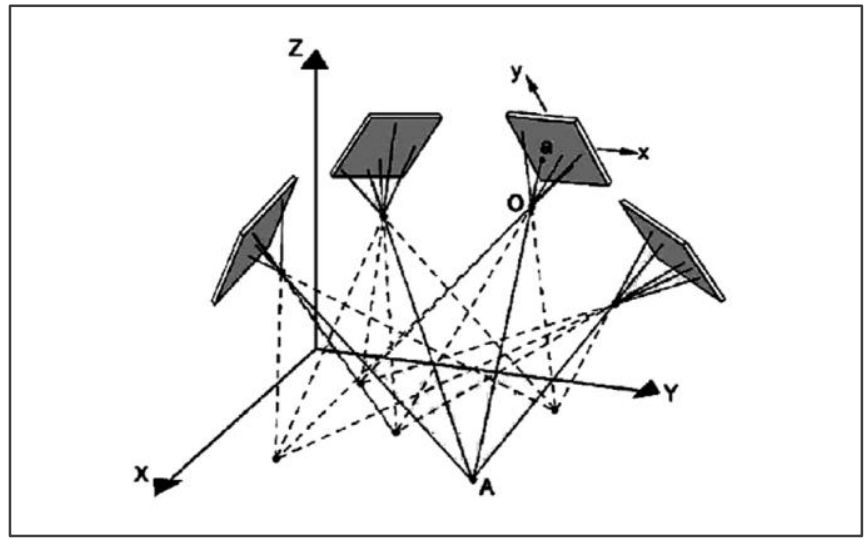

Figura 3: Princípio de funcionamento da fotogrametria (Cuypers et al., 2009).

Em muitos casos, no entanto, são necessários mais de dois pontos de vistas para reconstrução completa de um objeto complexo (Kasser \& Egels, 2002); da mesma forma como é preciso observar um objeto de diferentes ângulos para compreender inteiramente sua forma. Kasser e Egels (2002) apresentam duas possíveis estratégias para a aquisição de imagens de um objeto complexo; em configurações convergentes e paralelas (Figura 4).

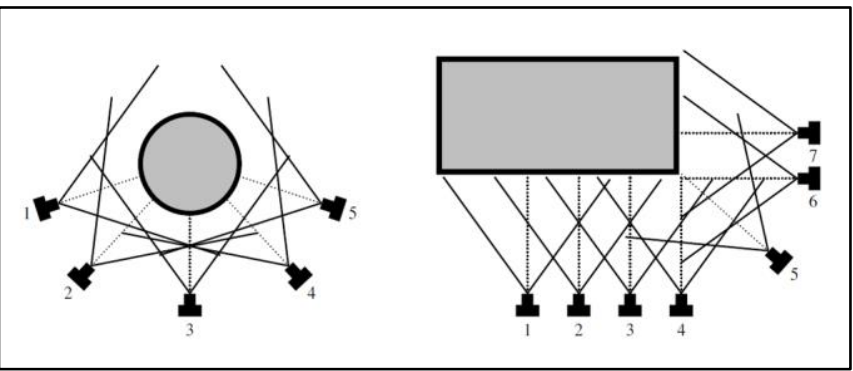

Figura 4: Estratégias para aquisição de conjuntos de imagens (Kasser \& Egels, 2002).

A orientação de múltiplas imagens e restituição das coordenadas dos objetos fotografados é um processo complexo cujos princípios matemáticos e algoritmos podem ser consultados em Kasser e Egels (2002), como também em Linder (2009). No entanto, apesar da natureza complexa da Fotogrametria, encontram-se, hoje, softwares e aplicativos voltados para o consumidor não especializado, onde este pode se preocupar apenas com a aquisição das imagens a serem processadas. Alguns destes aplicativos, por exemplo, 123D Catch, Recap 360 e Remake, além de serem gratuitos, oferecem ainda processamento em nuvem, isto é, o processamento é realizado em um servidor remoto, não dependendo do desempenho do computador do usuário.

O resultado possível de ser obtido com estas ferramentas, e com a fotogrametria, de modo geral, depende da qualidade das imagens adquiridas. Aplicam-se aqui requisitos gerais de fotografia. Iluminação, estabilização, tamanho e especificações do sensor influem na qualidade das imagens adquiridas e, consequentemente, na reconstrução tridimensional do objeto de interesse. Falando-se de Fotogrametria Digital (a partir de imagens adquiridas digitalmente), cabe salientar que um sensor com maior número de megapixels permite a aquisição de mais detalhes, aumentando também o tempo de processamento. A resolução de digitalização possível de ser atingida vai depender do número de megapixels disponíveis, da distância focal, e da distância entre objeto e câmera. Imagens com maior aproveitamento da área do sensor, com o objeto ou a área de interesse bem enquadrados, resultarão na aquisição de maiores detalhes da superfície.

Quanto ao objeto a ser digitalizado, também há de se tomar alguns cuidados; superfícies lisas, sem texturas distinguíveis, reflexivas, ou, até mesmo, com padrões repetidos podem dificultar o reconhecimento de pontos para a triangulação (Foster \& Halbstein, 2014; Kasser \& Egels, 2002). Esta característica - a necessidade de pontos de interesse para a triangulação -, pode, no entanto, ser explorada em situações em que se deseja uma configuração de aquisição convergente na qual a câmera permanece em uma posição fixa e o objeto gira em torno de um eixo vertical. Em uma situação normal a triangulação seria dificultada por elementos estáticos no fundo das imagens. Isto, no entanto, pode ser contornado posicionando um painel, ou fundo infinito, de cor neutra por trás do objeto, restringindo a detecção de pontos ao objeto.

No desenvolvimento do protótipo apresentado neste artigo, buscou-se aplicar estes princípios. Deste modo, foi possível otimizar o processo de aquisição das imagens.

\section{Metodologia}

\section{Prototipagem}

No livro de Richard Sennett 'The craftsman' (2008), o autor discorre sobre a contínua retroalimentação entre "a mão e a cabeça" nos trabalhos manuais. Sennett (2008) salienta também a natureza de imagens de possibilidade representada pelos sketches, onde "no processo de cristalização e refinamento destes à mão, o designer fica imerso e amadurece as ideias pensando neste processo de forma circular". O arquiteto, Renzo Piano, explica de forma semelhante o seu processo de trabalho: "Você começa com um esboço, então faz um desenho, daí você faz um modelo, depois vai para a realidade - vai a campo - e então você volta para o desenho. Assim, constrói-se uma circularidade entre desenhar e fazer, algo muito típico da abordagem do artesão" (Sennett, 2008). Estes princípios nortearam a metodologia utilizada neste trabalho.

As questões centrais aqui consistem na experiência tátil do desenhar, recortar, modelar com papelão ou outros materiais menos nobres, apenas como forma de representar possibilidades e auxiliar na absorção e desenvolvimento de um projeto; e também na presença da circularidade e iteração neste processo de tentativa-e-erro entre o conceito pensado e o teste da solução produzida. No 
desenvolvimento inicial do trabalho aqui exposto utilizou-se de técnicas manuais de desenho e modelagem (Figuras 5 e 6) para a criação e refinamento de alternativas. Em um primeiro momento ensaiou-se propostas para a configuração do dispositivo elaborando esboços bidimensionais, para então validar possíveis mecanismos através de protótipos confeccionados em papelão.

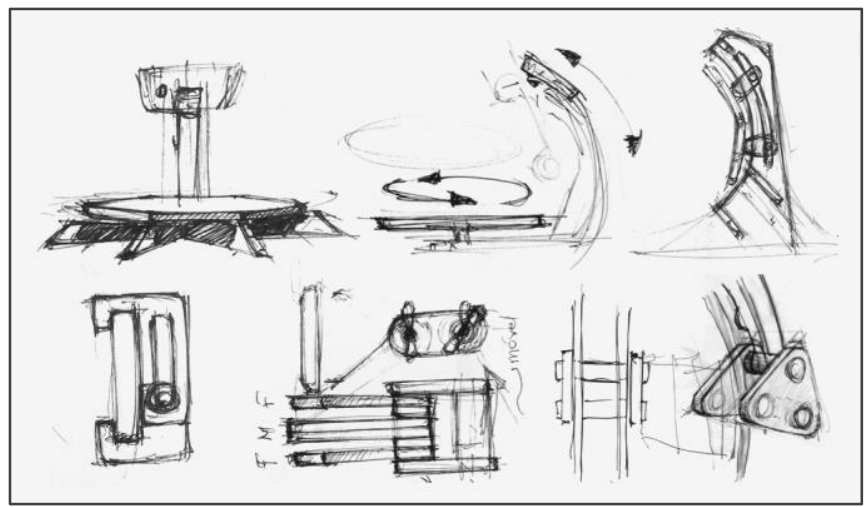

Figura 5: Rascunhos de exploração dos mecanismos de suporte do smartphone.

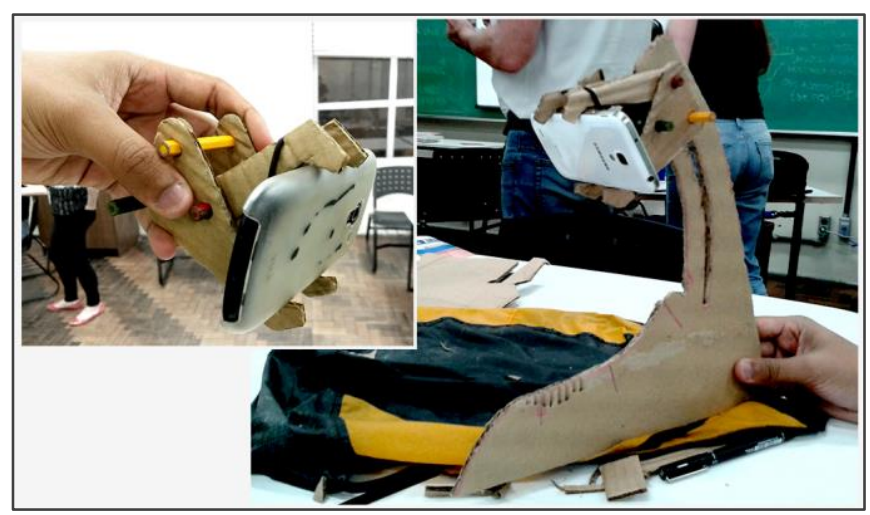

Figura 6: Modelagem preliminar em papelão da garra e da estrutura de suporte do smartphone.

Após estes estudos preliminares, digitalizaram-se as peças de papelão em um escâner 2D, e o desenvolvimento do projeto seguiu para o software CAD (Figura 7). As ferramentas oferecidas pelo software CAD asseguraram definição precisa de dimensões e angulações da mesa e da estrutura-suporte para o smartphone (Figura 8), permitindo ainda gerar o arquivo digital requerido para a sua fabricação na máquina de corte a laser.

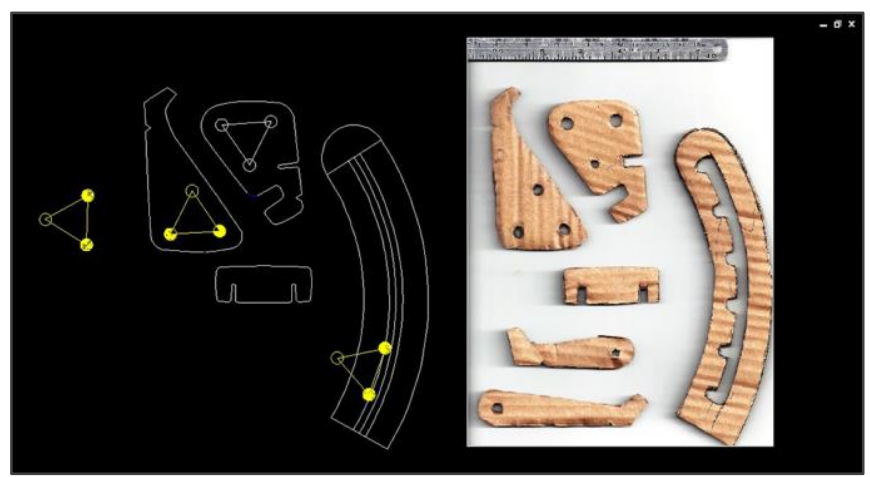

Figura 7: Desenho CAD das peças de papelão escaneadas.

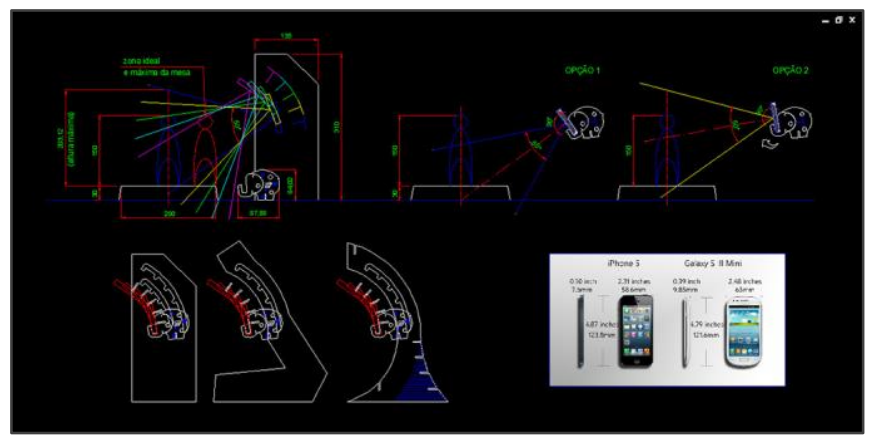

Figura 8: Dimensionamentos e angulações da mesa, da estrutura de suporte e campo de visão da câmera do smartphone.

A fabricação das peças realizou-se no maquinário disponível no LIFE/UFRGS. Com base nas primeiras estruturas montadas e os primeiros ensaios de funcionalidade, retornou-se ao software CAD, realizando aprimoramentos adicionais na configuração existente do dispositivo facilitador.

\section{Validação}

O dispositivo desenvolvido foi testado em conjunto com um smartphone Samsung Galaxy S4 Mini, lançado em 2013, com câmera de 8 megapixels. Smartphones com câmeras de resolução e especificações semelhantes podem ser encontrados no mercado a partir de $R \$ 500$. Para o processamento fotogramétrico das imagens foi utilizada a versão desktop do aplicativo gratuito $123 D$ Catch da Autodesk.

Para validar as vantagens inerentes à utilização do dispositivo facilitador desenvolvido, realizou-se um ensaio comparativo, analisando modelos tridimensionais obtidos por meio de sua utilização, e modelos provenientes de outros três procedimentos: Fotogrametria à mão livre e dois escâneres comerciais. Os modelos tridimensionais gerados foram analisados de forma qualitativa, avaliando o nível de detalhes de superfície adquiridos no processo de digitalização tridimensional.

O primeiro processo de digitalização tridimensional empregado na comparação foi a Fotogrametria à mão livre, isto é, com a aquisição de fotos manuseando o smartphone livremente em torno do objeto, sem fazer uso das estruturas de suporte e plataforma giratória. Foram utilizados o mesmo 
smartphone e aplicativo de fotogrametria que geraram o modelo de referência. Neste caso, sendo também baseado em Fotogrametria, analisou-se ainda a distribuição dos pontos de vistas das imagens adquiridas para triangulação.

Um segundo modelo comparativo foi obtido utilizando o escâner 3D comercial Artec EVA. Este dispositivo portátil realiza a digitalização tridimensional por emissão de luz branca estruturada, adquirindo até 2 milhões de pontos por segundo, junto de informações de cor do objeto. O equipamento possui alcance de 40 a $100 \mathrm{~cm}$, fácil operação e é acompanhado de uma solução de software para o processamento dos dados. Porém, é comercializado a US\$19,800.

Por último, utilizou-se um equipamento CNC Tecnodrill Digimill 3D com um cabeçote de digitalização Optimet ConoProbe 1000, que permite realizar digitalizações de alta precisão e resolução (Silva, 2011).

Cabe destacar que, com preço acima de $R \$ 150$ mil, o Digimill $3 D$ pode estar um pouco distante da realidade de projetos de baixo custo e Fab Labs. Ainda assim, o equipamento é utilizado neste estudo como referência dos resultados possíveis de serem obtidos com a digitalização tridimensional.

\section{Resultados}

O protótipo de dispositivo facilitador desenvolvido consiste em uma estrutura vertical e uma plataforma giratória. A estrutura vertical é o suporte do smartphone e permite o ajuste em cinco níveis de altura e inclinação. A plataforma gira livremente em torno de um eixo vertical, permitindo expor todos os lados do objeto à câmera do smartphone, sem que esta saia de sua posição. A plataforma giratória possui indicações de graduação, que servem de guia para adquirir uma quantidade específica de fotos, 16, 12 ou 8.

Uma vez que a plataforma e a estrutura vertical de suporte não estão conectadas fisicamente, é possível aproximá-las ou afastá-las a fim de melhorar o enquadramento do objeto posicionado sobre a plataforma. O protótipo foi dimensionado para a digitalização de objetos de até $15 \mathrm{~cm}$ de altura, assim, a estrutura vertical possui aproximadamente $34 \mathrm{~cm}$ de altura e a mesa $20 \mathrm{~cm}$ de diâmetro. Vindo ao encontro do que fora postulado, o dispositivo foi produzido utilizando papel Bismark (papel couro) de 2,5 mm de espessura, de forma a conferir rigidez e estabilidade ao conjunto, bem como possuir baixo custo de material.

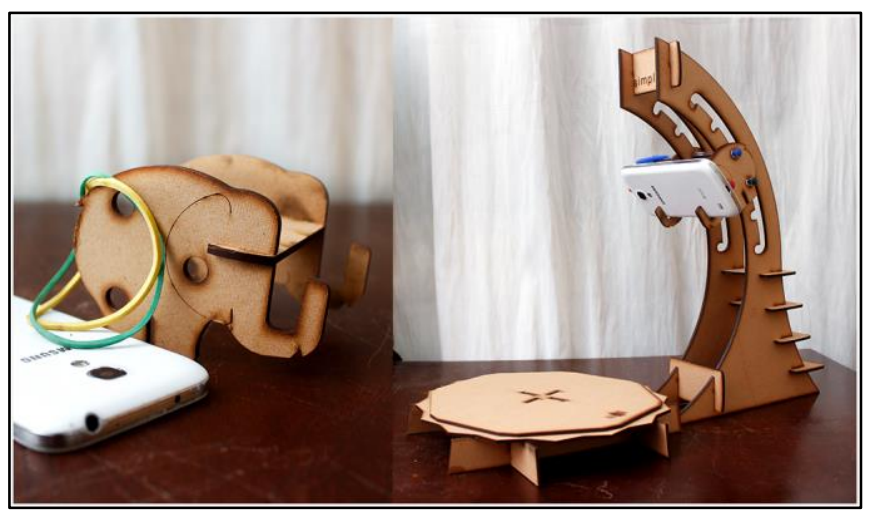

Figura 9: Suporte do smartphone e elásticos de segurança (esq) e estrutura completa montada com mesa giratória (dir).

O dispositivo foi denominado Simplify, em referência ao seu propósito de facilitar e simplificar o processo de aquisição de imagens para a fotogrametria. Além do smartphone utilizado no ensaio comparativo, o dispositivo suporta outros aparelhos com dimensões e posicionamento de câmera semelhantes sem maiores dificuldades. Ainda assim, a utilização de elásticos proporciona ainda maior fixação ao aparelho.

Com o objeto posicionado sobre a plataforma giratória, encaixa-se o smartphone no gancho do suporte, ajustando este na posição de altura e inclinação desejada para o enquadramento centralizado do objeto. O usuário pode, então, prosseguir para aquisição da primeira imagem, prestando atenção na marcação da posição inicial da plataforma.

Concluída a tomada de fotos em torno do objeto, é possível mudar o smartphone para outra posição do suporte vertical, adquirindo mais uma sequência de imagens de outra inclinação. Finalizada a aquisição de fotos, retira-se o smartphone do suporte para transferir as imagens para o computador, para posterior carregamento no software de processamento, neste caso o 123D Catch.

\section{Ensaio Comparativo}

Durante o ensaio comparativo, especificado na seção metodológica deste artigo, foi possível gerar três modelos tridimensionais completos do artefato de estudo, além de um recorte em alta resolução $(0,1 \mathrm{~mm})$ dos detalhes de sua superfície. Utilizou-se para isto um modelo decorativo de uma coruja, com aproximadamente $15 \mathrm{~cm}$ de altura.

Os dois procedimentos fotogramétricos realizados, com uso do Simplify e à mão livre, permitiram uma primeira análise centrada no processo de aquisição de imagens para processamento. A Figura 10 exibe o resultado da orientação externa das imagens adquiridas em ambos os casos. 


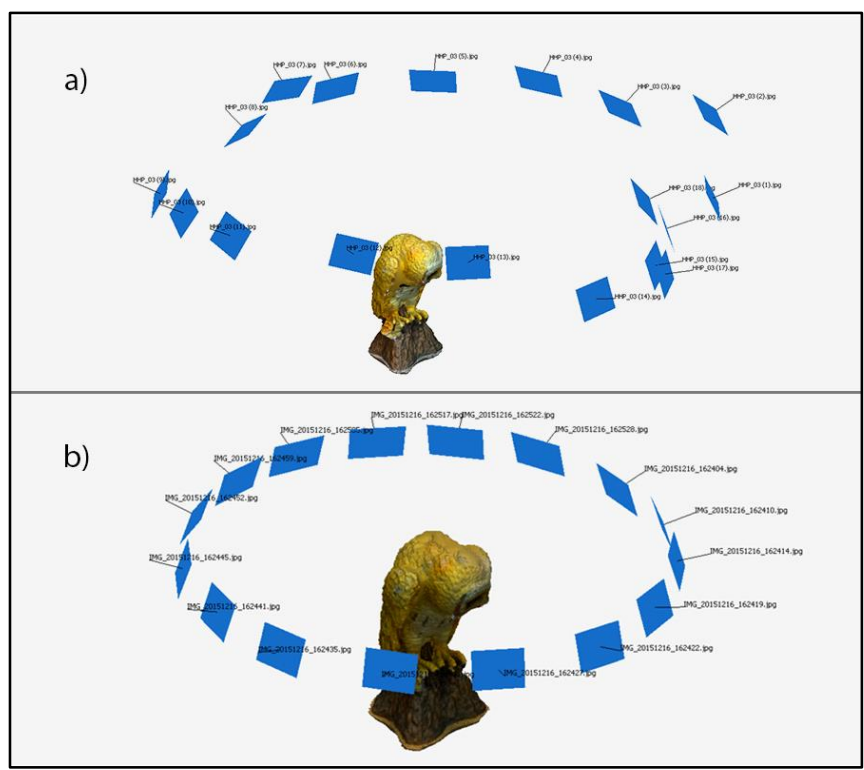

Figura 10: Orientação externa das fotografias tiradas para processamento. Fotogrametria sem Simplify (a) e fotogrametria com Simplify (b).

Como pode ser visto, a orientação externa das imagens adquiridas com o dispositivo apresenta uma distribuição uniforme em torno do objeto. A rotação do objeto em um eixo vertical, auxiliada pelas marcações gravadas na plataforma, não só assegura a distribuição homogênea das aquisições, como também preserva uma distância constante do objeto. $\mathrm{Na}$ aquisição de imagens à mão livre, foram tomadas 18 imagens. Com o Simplify, foram tomadas apenas 16, mas que proporcionaram melhor cobertura da superfície do objeto.

O efeito desta aquisição mais ordenada sobre a reconstrução tridimensional pode ser observado comparando as colunas a) e b) da Figura 11. Na linha superior observa-se, por exemplo, que as imagens tiradas à mão livre tiveram um pior resultado na reconstrução da geometria da área demarcada, que pode ser explicado pela distribuição irregular das aquisições em torno do objeto. Na linha de baixo, nota-se áreas de maior e menor resolução, em consequência da variação de distância do objeto e desfoques provocados pelo manejo manual da câmera.

Ainda na Figura 11, a coluna c) apresenta o resultado obtido com o Artec EVA. Nota-se que, apesar de ainda haver algumas falhas de reconstrução nas mesmas áreas analisadas anteriormente, o resultado obtido com o Simplify se aproxima deste escâner comercial.

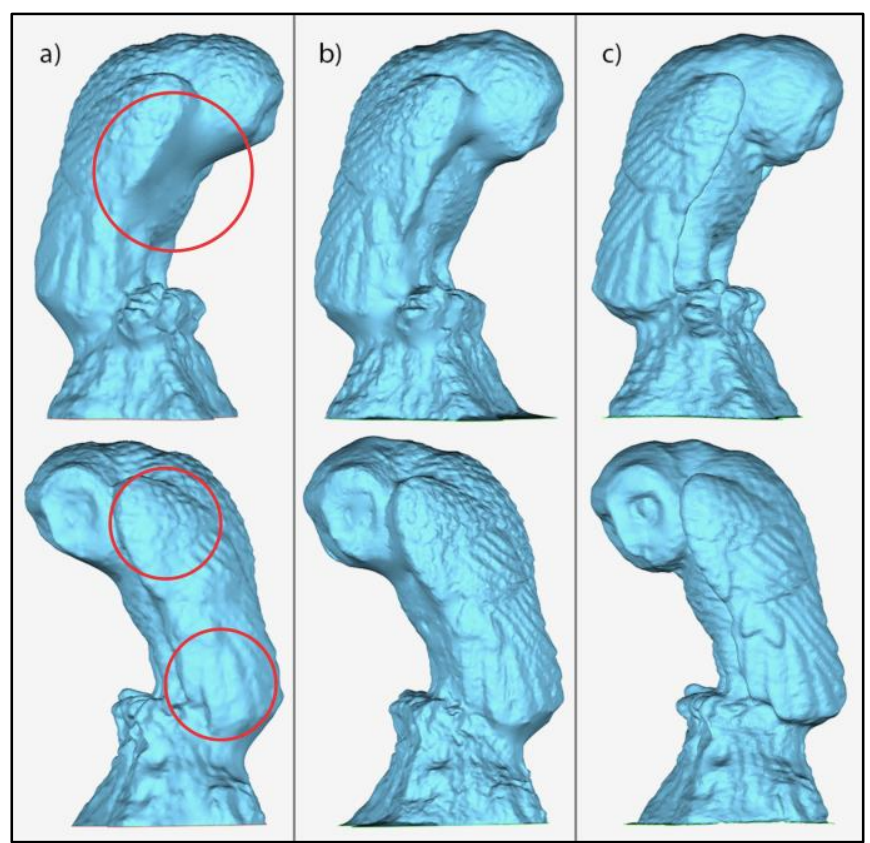

Figura 11: Modelo digitalizado. Fotogrametria sem Simplify (a), fotogrametria com Simplify (b) e Artec EVA (c).

Ampliando a análise comparativa, realizou-se ainda a digitalização de um recorte das costas da coruja, que apresenta geometria convexa simples com textura de penas detalhadas. Na Figura 12 observa-se que, apesar da dificuldade na reconstrução de geometrias com áreas de oclusão (Figura 11), a Fotogrametria com auxílio do Simplify (b) resultou em uma reconstrução tridimensional de maior resolução das penas do modelo, quando comparado à Fotogrametria à mão livre (a) e ao Artec EVA (c).

A Figura 12 exibe ainda uma digitalização, de alta precisão e resolução de $0,1 \mathrm{~mm}$, obtida com a Digimill $3 D(d)$. Cabe destacar que este equipamento foi utilizado apenas como referência, bem como sua resolução máxima pode ser bastante superior a essa.

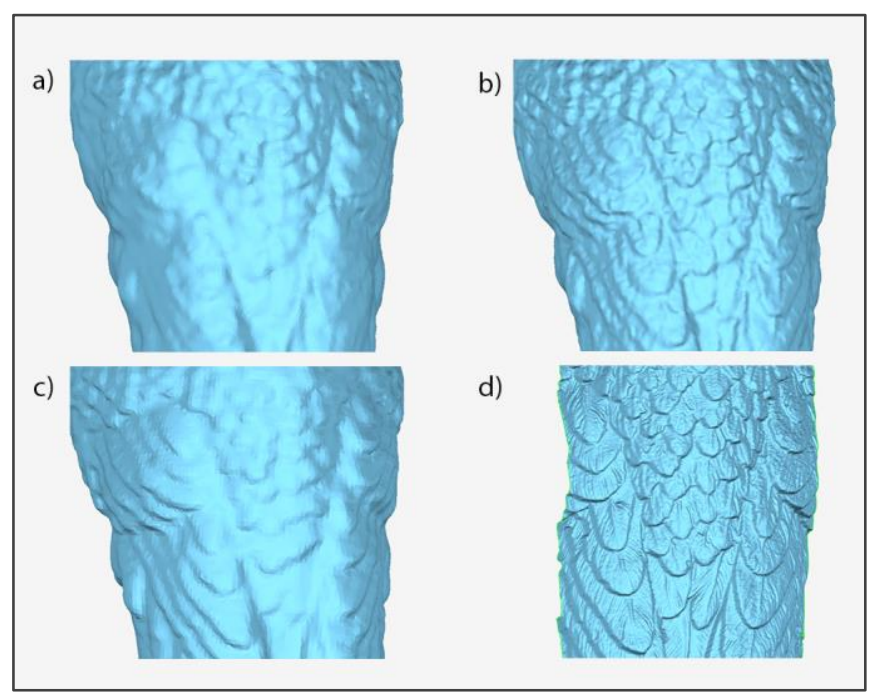


Figura 12: Recorte digitalizado. Fotogrametria à mão livre (a); Fotogrametria com Simplify (b); Artec EVA (c); e Digimill3D $(0,1 \mathrm{~mm})(\mathrm{d})$.

\section{Conclusão}

Com base nos resultados obtidos, pode-se concluir que o dispositivo desenvolvido, denominado Simplify, atende aos requisitos necessários à Fotogrametria identificados na revisão deste artigo. Além disso, cumpre o papel de facilitar o processo de aquisição de imagens para uma reconstrução tridimensional.

O ensaio comparativo mostrou que, apesar de o dispositivo desenvolvido não ser requisito para se realizar a digitalização tridimensional com smartphone, sua utilização pode resultar em uma digitalização de melhor qualidade. O resultado superior pode ser atribuído à distribuição uniforme das posições de aquisição - com indicação de graduação para realizar até 16 capturas ao redor do objeto -, e a manutenção de uma distância constante entre o objeto e a câmera.

Resultados semelhantes poderiam ser alcançados utilizando diferentes combinações de tripés e plataformas giratórias, no entanto, o protótipo apresentado se destaca por poder ser fabricado a partir de um único material de baixíssimo custo, além de ser montado por meio de encaixes e facilmente desmontado. Por exemplo, com uma chapa de tamanho padrão A0 $(1198 \times 841 \mathrm{~mm})$ de papel Bismark 2,5m comercializada a menos de $\mathrm{R} \$ 10,00$-, é possível produzir pelo menos 2 exemplares.

Para pesquisas futuras, o desenvolvimento do dispositivo reserva ainda possibilidades de automação da plataforma giratória e sincronização com o aplicativo de câmera do smartphone, a fim de realizar a aquisição automática a cada passo. Outra oportunidade consiste na parametrização do projeto, de forma a criar um sistema alimentado por medidas e parâmetros do smartphone a ser utilizado (largura, altura, posição e distância focal da câmera) para a otimização da configuração do desenho CAD do dispositivo e dos planos de corte.

Pensado para o contexto de Fab Labs e instituições de ensino, o dispositivo apresentado visa garantir a autonomia e sucesso de seus operadores, muitas vezes crianças e usuários não-especializados. Assim, acredita-se que o Simplify pode se tornar uma ferramenta para a difusão e democratização das tecnologias de digitalização tridimensional.

\section{Agradecimentos}

Agradecemos à Coordenação de Aperfeiçoamento de Pessoal de Nível Superior (CAPES) pela concessão das bolsas de estudo. Agradecemos também ao Laboratório de Design e Seleção de Materiais (LdSM) e ao Laboratório de Pesquisas em Fotogrametria (LAFOTO), ambos da UFRGS.

\section{Referências}

Cuypers, W., Van Gestel, N., Voet, A., Kruth, J.-P., Mingneau, J., \& Bleys, P. (2009). Optical measurement techniques for mobile and large-scale dimensional metrology. Optics and Lasers in $\begin{array}{ll}\text { Engineering, } & 47(3-4),\end{array}$ https://doi.org/10.1016/j.optlaseng.2008.03.013

Eulitz, M., \& Reiss, G. (2015). 3D reconstruction of SEM images by use of optical photogrammetry software. Journal of Structural Biology, 191(2), 190-196. https://doi.org/10.1016/j.jsb.2015.06.010

Evin, A., Souter, T., Hulme-Beaman, A., Ameen, C., Allen, R., Viacava, P., ... Dobney, K. (2016). The use of close-range photogrammetry in zooarchaeology: Creating accurate 3D models of wolf crania to study dog domestication. Journal of Archaeological Science: Reports, 9, 87-93. https://doi.org/10.1016/j.jasrep.2016.06.028

Eychenne, F., \& Neves, H. (2013). FAB LAB: A vanguarda da nova revolução industrial. São Paulo: Editorial Fab Lab Brasil.

Foster, S., \& Halbstein, D. (2014). Integrating 3D Modeling, Photogrammetry and Design. London: Springer London. Retrieved from http://link.springer.com/10.1007/978-1-44716329-9

Gershenfeld, N. (2006). Unleash your creativity in a Fab Lab. Retrieved from http://www.ted.com/talks/neil_gershenfeld_on_fab_labs?languag $\mathrm{e}=\mathrm{en}$

Gershenfeld, N. (2012). How to make almost anything: The digital fabrication revolution. Foreign Aff., 91, 43.

Kasser, M., \& Egels, Y. (2002). Digital photogrammetry. London; New York: Taylor \& Francis. Retrieved from http://site.ebrary.com/id/5003410

Linder, W. (2009). Digital Photogrammetry. Berlin, Heidelberg: Springer Berlin Heidelberg. Retrieved from http://link.springer.com/10.1007/978-3-540-92725-9

Lipson, H., \& Kurman, M. (2013). Fabricated: the new world of $3 D$ printing. Indianapolis, Indiana: John Wiley \& Sons.

Martínez, S., Ortiz, J., \& Gil, M. (2015). Geometric documentation of historical pavements using automated digital photogrammetry and high-density reconstruction algorithms. Journal of Archaeological Science, 53, https://doi.org/10.1016/j.jas.2014.10.003

Pesce, M., Galantucci, L. M., Percoco, G., \& Lavecchia, F. (2015). A Low-cost Multi Camera 3D Scanning System for Quality Measurement of Non-static Subjects. Procedia CIRP, 28, 88-93. https://doi.org/10.1016/j.procir.2015.04.015

Redweik, P. (2013). Photogrammetry. In Sciences of Geodesy - II: Innovations and Future Developments (pp. 133-183). Berlin, Heidelberg: Springer Berlin Heidelberg.

Sennett, R. (2008). The craftsman. New Haven: Yale University Press.

Silva, F. P. da. (2011). Usinagem de espumas de poliuretano e digitalização tridimensional para fabricação de assentos personalizados para pessoas com deficiência (Tese de Doutorado em Engenharia de Minas, Metalúrgica e de Materiais). Universidade Federal do Rio Grande do Sul, Porto Alegre. Retrieved from http://www.lume.ufrgs.br/handle/10183/36040

Tang, C. H. H., Tang, H. E., \& Tay, P. K. J. (2016). Low cost digital close range photogrammetric measurement of an as-built anchor handling tug hull. Ocean Engineering, 119, 67-74. https://doi.org/10.1016/j.oceaneng.2016.04.016 
SIGraDi 2016, XX Congress of the Iberoamerican Society of Digital Graphics

9-11, November, 2016 - Buenos Aires, Argentina

Tommaselli, A. M. G., Silva, J. F. C., Hasegawa, J. K., Galo, M., \& Dal Poz, A. P. (1999). Fotogrametria: aplicações a curta distância. In FCT (Vol. 40, pp. 147-59).

Urbanová, P., Hejna, P., \& Jurda, M. (2015). Testing photogrammetry-based techniques for three-dimensional surface

$\begin{aligned} & \text { documentation in forensic pathology. Forensic } \\ & 250,\end{aligned}$
International,
https://doi.org/10.1016/j.forsciint.2015.03.005
$77-86$.

